\title{
Uso Off-Label de Stent Farmacológico: Eficácia versus Efetividade
} Ver artigo relacionado
na página 31

\author{
Fábio Sândoli de Brito Jr. ${ }^{1}$, Adriano Caixeta ${ }^{2}$
}

E 2003 e 2004, o Food and Drug Administration (FDA), órgão governamental americano que controla o setor de alimentos e remédios nos Estados Unidos, aprovou para uso clínico os dois primeiros stents farmacológicos, Cypher ${ }^{\mathrm{TM}}$ (Cordis Corporation Warren, Estados Unidos) e Taxus ${ }^{\mathrm{TM}}$ (Boston Scientific - Natick, Estados Unidos), eluidores de sirolimus e paclitaxel, respectivamente. A decisão do FDA baseouse nos excelentes resultados de estudos clínicos randomizados de fase III (SIRIUS e TAXUS IV) comparando o stent farmacológico a seu equivalente stent convencional, em condições de baixa ou de moderada complexidade clínica e anatômica, registradas nas bulas desses dispositivos e conhecidas como indicações on-label. Mais recentemente, a apresentação dos resultados dos estudos randomizados ENDEAVOR IV e SPIRIT III, também com critérios de inclusão pouco complexos, levaram, em 2008, à aprovação para uso clínico nos Estados Unidos dos stents Endeavor ${ }^{\mathrm{TM}}$ (Medtronic Santa Rosa, Estados Unidos) e Xience VTM/Promus (Abbott Vascular - Abbott Park, Estados Unidos/Boston Scientific - Natick, Estados Unidos), eluidores de zotarolimus e everolimus, respectivamente. No Brasil, nos países europeus e em diversos outros países do mundo, os resultados de outros estudos preliminares demonstrando a superioridade dos stents farmacológicos em prevenir a reestenose, também realizados em situações de baixa complexidade, foram críticos para sua aprovação e rápida incorporação com, pelo menos, um ano de antecedência em relação ao FDA. De fato, dada a expressiva redução de eventos clínicos relacionados à reestenose, a confiança no uso dos stents farmacológicos aumentou e os cardiologistas, extrapolando os resultados dos estudos randomizados iniciais, rapidamente expandiram as indicações desses dispositivos para condições mais complexas, não previstas na bula do fabricante, denominadas off-label. Nesse grupo, incluem-se pacientes multiarteriais, com infarto do miocárdio, lesões longas (> $30 \mathrm{~mm}$ ), vasos de muito pequeno calibre $(<2,5 \mathrm{~mm})$, reestenoses intrastent, bifurcações, lesões com trombo e lesões em enxertos de safena, entre outras. As indicações offlabel, já há vários anos, correspondem a mais de dois terços das indicações de stents farmacológicos, como no estudo de Costa et al. ${ }^{1}$, publicado nesta edição da Revista Brasileira de Cardiologia Invasiva. Esse fenômeno paradoxal - o número de implantes de stents farmacológicos proporcionalmente maior em indicações off-label comparado às indicações on-label - fica ainda mais evidente quando existem restrições de ordem econômica, como em nosso País. Nessas circunstâncias, reservam-se os stents farmacológicos justamente para as condições de maior complexidade e de maior risco de reestenose (off-label). Diferentemente, nas indicações on-label, em que existe aprovação formal para o uso do stent farmacológico, empregam-se, frequentemente, os stents convencionais, presumindo desempenho aceitável.

É importante ressaltar que a indicação preferencial para o uso dos stents farmacológicos em situações off-label ocorre de forma individualizada e baseia-se em consenso de especialistas, que avaliam ser esse o tratamento com a maior probabilidade de sucesso terapêutico a longo prazo, situações nas quais, sabidamente, o desempenho clínico do stent convencional é inferior. Evidentemente, não se espera que os stents farmacológicos tenham, nas situações off-label, desempenho semelhante àquele obtido nas indicações onlabel. É natural que os resultados angiográficos e clínicos em situações off-label sejam inferiores, conforme demonstraram alguns registros de mundo real ${ }^{2-4} \mathrm{e}$, também, o de Costa et al. ${ }^{1}$. Esses resultados inferiores nas situações off-label devem-se não ao desempenho do dispositivo per se, mas sim às próprias condições de apresentação da doença aterosclerótica e comorbidades, como a presença de diabetes ou insuficiên-

\footnotetext{
1 Hospital Israelita Albert Einstein - São Paulo, SP, Brasil.

2 Columbia University Medical Center e Cardiovascular Research Foundation - New York, NY, Estados Unidos.

Correspondência: Fábio Sândoli de Brito Jr. Rua Dom Armando Lombardi, 819/82-A - Jardim Guedala - São Paulo, SP, Brasil -

CEP 05616-011

E-mail: fsbrito@einstein.br

Recebido em: 16/3/2009 - Aceito em: 18/3/2009
} 
cia renal, por exemplo. Os stents convencionais também apresentam piores resultados nas situações offlabel, comparativamente às situações on-label. Por isso, faz-se necessário analisar comparativamente os desempenhos dos stents farmacológicos e convencionais, exclusivamente para situações off-label. Nesse caso, as evidências indicam que o emprego dos stents farmacológicos se associa a taxas de morte e infarto que são, no mínimo, equivalentes às obtidas com os stents convencionais, com a clara vantagem de oferecer enorme redução da ocorrência de reestenose clínica $^{5,6}$. Isso atenua os questionamentos e as incertezas relativos à segurança desses dispositivos, principalmente no que se refere ao aumento da incidência de trombose tardia quando de seu emprego em situações off-label. Adicionalmente, cabe destacar que, nos últimos anos, inúmeros estudos randomizados demonstraram a superioridade dos stents farmacológicos em situações consideradas off-label, como lesões longas (com ou sem overlap de stents) ${ }^{7}$, reestenose intrastent ${ }^{8}$, oclusões crônicas $^{9}$ e infarto agudo do miocárdio ${ }^{10}$.

Por fim, vale lembrar que, nessa controvérsia sobre o emprego de drogas ou dispositivos médicos em situações off-label, sempre há diversos atores envolvidos: a indústria, as fontes pagadoras, os pacientes, o cardiologista clínico e o intervencionista. Nesse sentido, o estudo de Costa et al. ${ }^{1}$, demonstrando, após 2,7 anos de seguimento clínico médio, a segurança e a eficácia dos stents farmacológicos em uma população não selecionada, apoia a decisão que mais frequentemente tem sido tomada pelos intervencionistas na prática clínica e contribui para consolidar o uso dos stents farmacológicos em situações off-label. No entanto, o estudo de Costa et al. ${ }^{1}$ apresenta algumas limitações que requerem cuidadosa interpretação. Tratase de um estudo observacional não controlado e, portanto, suscetível a vieses inerentes à inabilidade de controle de numerosos fatores de confusão. Adicionalmente, o estudo não possui poder estatístico suficiente para analisar variáveis clínicas isoladas e infrequentes, como morte cardíaca e trombose de stent. Por fim, foram excluídos do estudo pacientes na fase aguda do infarto e lesões em enxertos de safena, condições nas quais as taxas de eventos adversos são maiores. Assim, esses seus resultados não podem ser extrapolados e não são representativos de todas as indicações off-label. De qualquer forma, o estudo de Costa et al. ${ }^{1}$ reforça os dados da literatura e tem o grande mérito de confirmar que os stents farmacológicos, comprovadamente eficazes, são, também, efeti- vos, ou seja, eficazes em condições reais da prática clínica diária e não apenas no cenário ideal do estudo randomizado.

\section{CONFLITO DE INTERESSES}

Os autores declararam inexistência de conflito de interesses.

\section{REFERÊNCIAS BIBLIOGRÁFICAS}

1. Costa Jr JR, Sousa AGMR, Costa R, Moreira A, Cano M, Maldonado G, et al. Até que ponto a complexidade angiográfica influi nos resultados tardios de pacientes tratados com stents farmacológicos? Comparação entre indicações on label e off label no Registro DESIRE. Rev Bras Cardiol Invas. 2009;17(1):31-8.

2. Beohar N, Davidson CJ, Kip KE, Goodreau L, Vlachos HA, Meyers $\mathrm{SN}$, et al. Outcomes and complications associated with off-label and untested use of drug-eluting stents. JAMA. 2007;297(18):1992-2000.

3. Rao SV, Shaw RE, Brindis RG, Klein LW, Weintraub WS, Peterson ED. On- versus off-label use of drug-eluting coronary stents in clinical practice (report from the American College of Cardiology National Cardiovascular Data Registry [NCDR]). Am J Cardiol. 2006;97(10):1478-81.

4. Win HK, Caldera AE, Maresh K, Lopez J, Rihal CS, Parikh $M A$, et al. Clinical outcomes and stent thrombosis following off-label use of drug-eluting stents. JAMA. 2007;297(18): 2001-9.

5. Applegate RJ, Sacrinty MT, Kutcher MA, Santos RM, Gandhi SK, Baki TT, et al. "Off-label" stent therapy 2-year comparison of drug-eluting versus bare-metal stents. J Am Coll Cardiol. 2008;51(6):607-14.

6. Marroquin OC, Selzer F, Mulukutla SR, Williams DO, Vlachos HA, Wilensky RL, et al. A comparison of bare-metal and drug-eluting stents for off-label indications. $N$ Engl J Med. 2008;358(4):342-52.

7. Grube E, Dawkins KD, Guagliumi G, Banning AP, Zmudka K, Colombo A, et al. TAXUS VI 2-year follow-up: randomized comparison of polymer-based paclitaxel-eluting with bare metal stents for treatment of long, complex lesions. Eur Heart J. $2007 ; 28(21): 2578-82$.

8. Dibra A, Kastrati A, Alfonso F, Seyfarth M, Pérez-Vizcayno MJ, Mehilli J, et al. Effectiveness of drug-eluting stents in patients with bare-metal in-stent restenosis: meta-analysis of randomized trials. J Am Coll Cardiol. 2007;49(5):616-23.

9. Suttorp MJ, Laarman GJ, Rahel BM, Kelder JC, Bosschaert MA, Kiemeneij F, et al. Primary Stenting of Totally Occluded Native Coronary Arteries II (PRISON II): a randomized comparison of bare metal stent implantation with sirolimuseluting stent implantation for the treatment of total coronary occlusions. Circulation. 2006;114(9):921-8.

10. Kastrati A, Dibra A, Spaulding C, Laarman GJ, Menichelli M, Valgimigli $M$, et al. Meta-analysis of randomized trials on drug-eluting stents vs. bare-metal stents in patients with acute myocardial infarction. Eur Heart J. 2007;28(22):2706-13. 